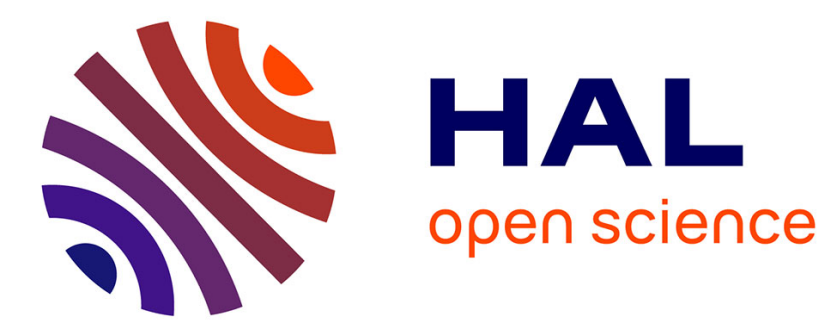

\title{
Propriétés spectroscopiques de U4+ dans ThBr4
}

P. Delamoye, S. Hubert, M. Hussonnois, J. Krupa, M. Genet, R. Guillaumont, N. Edelstein, J. Conway

\section{To cite this version:}

P. Delamoye, S. Hubert, M. Hussonnois, J. Krupa, M. Genet, et al.. Propriétés spectroscopiques de U4+ dans ThBr4. International Conference On Electronic Structure Of The Actinides 3, 1978, Grenoble, France. pp.C4-173-C4-175, 10.1051/jphyscol:1979454 . jpa-00218850

\section{HAL Id: jpa-00218850 https://hal.science/jpa-00218850}

Submitted on 1 Jan 1979

HAL is a multi-disciplinary open access archive for the deposit and dissemination of scientific research documents, whether they are published or not. The documents may come from teaching and research institutions in France or abroad, or from public or private research centers.
L'archive ouverte pluridisciplinaire HAL, est destinée au dépôt et à la diffusion de documents scientifiques de niveau recherche, publiés ou non, émanant des établissements d'enseignement et de recherche français ou étrangers, des laboratoires publics ou privés. 


\title{
Propriétés spectroscopiques de $\mathrm{U}^{4+}$ dans $\mathrm{ThBr}_{4}$
}

\author{
P. Delamoye, S. Hubert, M. Hussonnois, J. C. Krupa, M. Genet, R. Guillaumont, N. Edelstein (*) \\ et J. Conway (*)
}

\begin{abstract}
Laboratoire de Radiochimie, Institut de Physique Nucléaire, Université de Paris-Sud, B.P. N ${ }^{\circ} 1,91406$ Orsay, France (*) Materials and Molecular Research Division, Lawrence Berkeley Laboratory, University of California Berkeley, California 94720 , U.S.A.
\end{abstract}

\begin{abstract}
Résumé. - L'indexation de raies à zéro phonon du spectre d'absorption de $\mathrm{ThBr}_{4}: \mathrm{U}^{4+}$ est discutée, compte tenu du spectre de vibration de la matrice et des spectres d'émission de ces monocristaux excités par laser.
\end{abstract}

\begin{abstract}
Assignment of zero phonon line in $\mathrm{ThBr}_{4}: \mathrm{U}^{4+}$ absorption spectrum is discussed according to vibrational spectra of $\mathrm{ThBr}_{4}$ matrix and laser induced emission spectra of the doped single crystal.
\end{abstract}

Les spectres d'absorption $\alpha, \sigma, \pi$, relevés à $4 \mathrm{~K}$ sur des monocristaux de $\mathrm{ThBr}_{4}: \mathrm{U}^{4+}$ montrent que toutes les transitions observées entre 4200 et $22000 \mathrm{~cm}^{-1}$ sont d'origine dipolaire électrique [1] $\mathrm{Si} \mathrm{U}^{4+}$ remplace dans la matrice de $\mathrm{ThBr}_{4}\left(\mathrm{D}_{4 \mathrm{~h}}^{19}\right.$ $I_{4_{1}} /$ amd) un ion $\mathrm{Th}^{4+}$, il occupe un site de symétrie $\mathrm{D}_{2 \mathrm{~d}}$. Le spectre de $\mathrm{U}^{4+}$ a été indexé dans cette hypothèse essentiellement en sélectionnant les raies les plus intenses comme des transitions à zéro phonon [1]. Toutefois, l'examen critique de l'ensemble des interprétations des spectres d'absorption de $\mathrm{U}^{4+}$, notamment dans la symétrie $\mathrm{D}_{2 \mathrm{~d}}$ [2-3], même lorsqu'on possède des données complémentaires aux données d'absorption, montre que la sélection des transitions purement électroniques n'a jamais été parfaitement rigoureuse. Or, la nature des monocristaux de $\mathrm{ThBr}_{4}: \mathrm{U}^{4+}$ permet d'obtenir des informations originales pour améliorer l'indexation des spectres. En effet, $\mathrm{U}^{4+}$ et $\mathrm{Th}^{4+}$ ayant des masses voisines, les modes de vibration de $\mathrm{U}^{4+}$ seront ceux de la matrice et, comme nous l'avons montré, $\mathrm{U}^{4+}$ fluoresce dans $\mathrm{ThBr}_{4}[1]$.

Nous avons donc entrepris la mesure des fréquences des modes normaux de vibration de $\mathrm{ThBr}_{4}$ et relevé des spectres d'émission de $\mathrm{U}^{4+}$ obtenus en particulier par excitation sélective de cet ion. Nous rapportons ici les résultats définitivement acquis.

1. Spectre d'absorption de $\operatorname{ThBr}_{4}: \mathrm{U}^{4+}$. - Nous avons vérifié que le spectre d'absorption de $\mathrm{ThBr}_{4}$ : $\mathrm{U}^{4+}$ à $4 \mathrm{~K}$ était bien celui de $\mathrm{U}^{4+}$ et que l'étalonnage des spectres était correct dans l'infra-rouge. Pour cela, plusieurs monocristaux de $\mathrm{ThBr}_{4}$ dopés à $1 \%$ et obtenus avec $\mathrm{UBr}_{4}$ ou $\mathrm{UO}_{2}$ ont été soumis à analyse avec divers appareils. Les spectres sont analogues, à un décalage près en longueur d'onde, à ceux de $\mathrm{ThCl}_{4}$ : $\mathrm{U}^{4+}$ et $\mathrm{Na}_{4}\left[\mathrm{Th}\left(\mathrm{C}_{6} \mathrm{H}_{4} \mathrm{O}_{2}\right)_{4}\right] 21 \mathrm{H}_{2} \mathrm{O}$ : $\mathrm{U}^{4+}$ [4]. C'est sur les données complètes des spec- tres $\sigma$ et $\pi$ relevés à $4 \mathrm{~K}$ [5] que nous discuterons. Nous avons vérifié que la structure des cristaux $\mathrm{ThBr}_{4}: \mathrm{U}^{4+}$ est la même que celle de $\mathrm{ThBr}_{4}$.

2. Modes normaux de vibration de $\mathbf{T h B r}_{4} \cdot-$ L'ensemble des résultats obtenus par diffusion Raman et absorption infra-rouge est reporté sur le tableau I. Il n'y a pas de problèmes pour indexer les dix modes normaux actifs en Raman. En revanche, devant l'impossibilité de préparer des lames de $\mathrm{ThBr}_{4}$ d'épaisseur inférieure à $0,3 \mathrm{~mm}$, les spectres IR ont été relevés sur une couche mince de microcristaux. On ne peut alors distinguer entre spectre $\sigma\left(\mathrm{E}_{\mathrm{v}}\right)$ et $\pi\left(A_{2 u}\right)$. L'indexation portée sur le tableau $I$ a été réalisée, compte tenu de la corrélation $T_{d}-D_{4 h}$. Le mode $\nu\left(\mathrm{T}_{2}\right)$ de $\mathrm{T}_{\mathrm{d}}$, qui a toujours la fréquence la plus élevée [6], doit conduire à des modes $B_{2 \varepsilon}, A_{2 u}, E_{u}$ et $E_{g}$ de fréquences proches. En conséquence, la bande vers $155 \mathrm{~cm}^{-1}$ très large serait due aux modes $E_{v}+$ $A_{2 u}$, non résolus $\left(\sim 160\right.$ et $\left.155 \mathrm{~cm}^{-1}\right)$. Il faut qu'un mode $A_{2 u}$ soit proche d'un $B_{2 g}$ : nous avons donc choisi celui à $70 \mathrm{~cm}^{-1}$. Les spectres de réflexion $\sigma$ et $\pi$ sont en cours de dépouillement pour lever l'incertitude de l'indexation des niveaux actifs en infrarouge.

Tableau I.

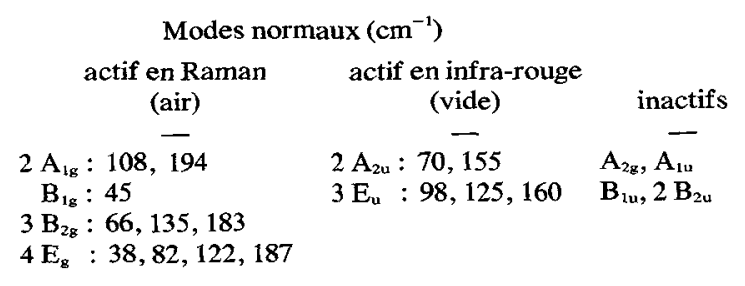

3. Spectres d'émission de $\mathbf{T h B r}_{4}: \mathrm{U}^{4+}$. - L'excitation de $\mathrm{ThBr}_{4}: \mathrm{U}^{4+}$ entre 5160 et $5770 \AA$ avec une 
lumière laser définie à $0,6 \AA$ près conduit à divers spectres d'émission que nous avons relevés dans le visible. Ils présentent tous des raies intenses accompagnées de raies vibroniques dont la position correspond généralement bien aux fréquences des modes normaux que nous avons mesurés. Par exemple, l'émission provoquée par excitation à 5166 ou $5175 \AA$ donne une raie intense à $5182 \AA$ (19 $292 \mathrm{~cm}^{-1}$ ), suivie de 7 raies peu intenses à +72 , $91,103,115,145,169$ et $182 \mathrm{~cm}^{-1}$. Ces expériences permettent de distinguer deux types de raies d'absorption. La différenciation repose sur des phénomènes complexes que nous ne pouvons discuter ici. Les raies de chaque type, 1 ou 2 , sont telles qu'elles alimentent des spectres d'émission différents, dont les raies principales sont séparées par l'énergie d'un phonon. Chaque raie du type 2 semble toujours associée avec une raie de type 1 pour former un couple, $\sigma$ ou $\pi$ (Fig. 1).

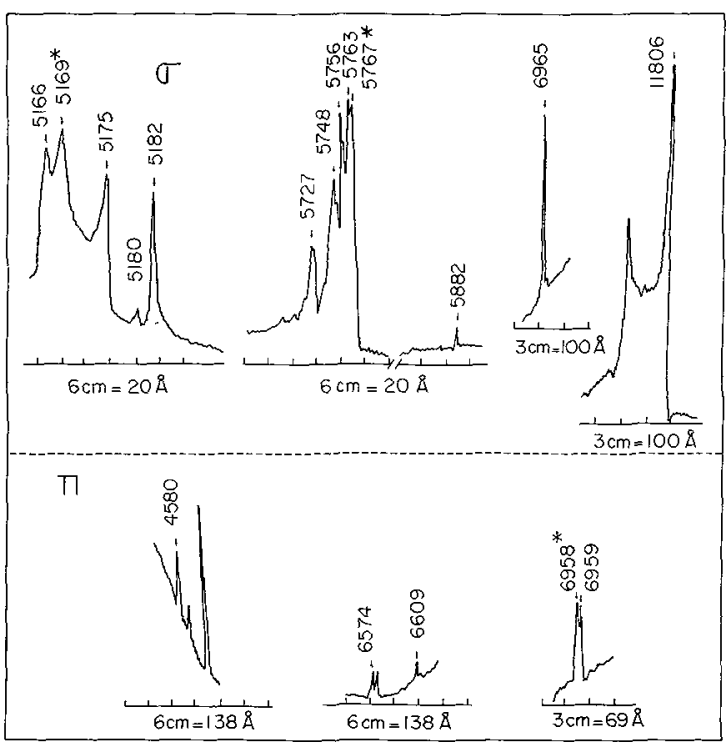

Fig. 1. - Raies d'absorption $\sigma$ et $\pi$ de $\mathrm{ThBr}_{4}: \mathrm{U}^{4+}$ discutées dans le tableau II.

* Raies attribuées au type 2 .

Sur le tableau $I I$ on a reporté :

- les longueurs d'onde des raies de type 1 communes à des spectres d'émission et au spectre d'absorption, actuellement identifiés par excitation sélective. Pour ce qui concerne l'émission, il s'agit de raies intenses;

- les longueurs d'onde de raies d'absorption, probablement de type 1 , car très fines, qui coïncident avec une raie des spectres d'émission obtenus par excitation fixe à $3363 \AA$ et à $5145 \AA$.

Toutes ces raies sont à zéro phonon.

Sur le tableau II apparaissent aussi les longueurs d'onde de la lumière laser correspondant à une raie du spectre d'absorption.

On remarquera le rôle particulier de l'état à
Tableau II.

\begin{tabular}{|c|c|c|}
\hline 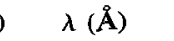 & 2) & $\lambda(\AA)$ \\
\hline $5180(\sigma)$ & $4580(\pi)$ & - \\
\hline $5182(\alpha, \sigma)$ & 3363 & $5166(\alpha, \sigma), 5175(\alpha, \sigma)$ \\
\hline $5882(\alpha, \sigma)$ & $5756(\alpha, \sigma)$ & $5763(\sigma)$ \\
\hline $6574(\pi)$ & $\begin{array}{l}5166(\alpha, \sigma) \\
5182(\alpha, \sigma)\end{array}$ & $5175(\alpha, \sigma), 5180(\sigma)$ \\
\hline $6609(\pi)$ & $\begin{array}{l}5166(\alpha, \sigma) \\
5182(\alpha, \sigma)\end{array}$ & $5175(\alpha, \sigma), 5180(\sigma)$ \\
\hline $6959(\pi)$ & $5175(\alpha, \sigma)$ & $5756(\alpha, \sigma), 5763(\sigma)$ \\
\hline $6965(\alpha, \sigma)$ & $\begin{array}{l}5145 \\
5180(\sigma) \\
5182(\alpha, \sigma)\end{array}$ & $\begin{array}{l}5166(\alpha, \sigma), 5175(\alpha, \sigma), \\
5756(\alpha, \sigma), 5763(\sigma)\end{array}$ \\
\hline $806(\alpha$, & 5145 & \\
\hline
\end{tabular}

Colonne 1 : raies en coïncidence dans les spectres d'émission et le spectre d'absorption.

Colonne 2 : niveaux d'absorption excités par laser qui alimentent les niveaux purement électroniques indiqués colonne 1 .

$6965 \AA$ comme état relais. Il se situe dans le domaine d'énergie où on attend l'état ${ }^{3} \mathrm{P}_{0}$, particulièrement intéressant puisqu'il n'est pas dégénéré. Le profil des raies indiquées tableau II apparaît sur la figure 1.

L'identification d'autres raies à zéro phonon par cette méthode d'investigation est en cours.

4. Raies vibroniques associées à une raie à zéro phonon. - Si l'état fondamental de $\mathrm{U}^{4+}$ en symétrie $\mathrm{D}_{2 \mathrm{~d}}$ est $\Gamma_{4}$, les transitions à zéro phonon $\Gamma_{1}$ apparaissent seulement sur le spectre $\pi$ et les $\Gamma_{5}$ seulement sur le spectre $\sigma$. Dans tous les autres cas, les transitions sont vibroniques.

Les règles de sélection courantes permettent de prévoir qu'à une transition $\Gamma_{1}$ sont associées des transitions vibroniques à un phonon $\mathrm{A}_{1 \mathrm{~g}}+\mathrm{B}_{1 \mathrm{u}}$ pour le spectre $\pi$ et $\mathrm{E}_{\mathrm{g}}+\mathrm{E}_{\mathrm{u}}$ pour le spectre $\sigma$. Les structures vibroniques à un phonon associées aux transitions $\Gamma_{4}-\Gamma_{5}$ sont plus complexes. En effet, pour les spectres $\pi$, ce sont les modes $E_{g}+E_{u}$ qui lèvent

Tableau III.

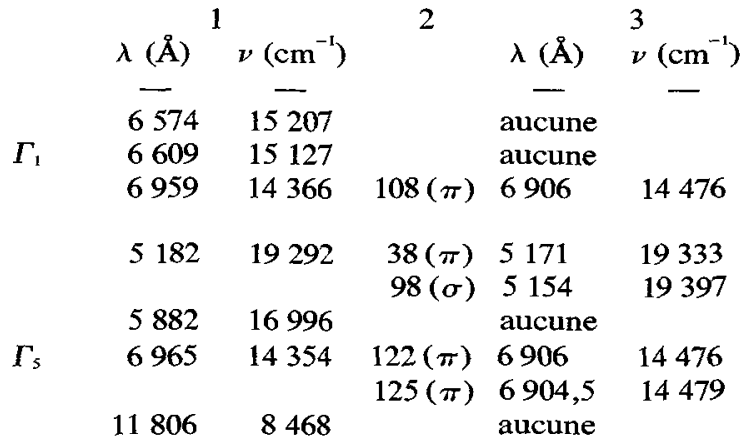

Longueurs d'onde et énergies (col. 1) des transitions à zéro phonon $\Gamma_{1}$ du spectre $\pi$ et $\Gamma_{5}$ du spectre $\sigma$ auxquelles sont associées les transitions vibroniques (col. 3) des spectres $\pi$ et $\sigma$. Les énergies des modes mis en cause apparaissent (col. 2). 
l'interdiction et pour les spectres $\sigma$ les niveaux vibrationnels susceptibles d'être atteints sont :

$$
A_{1 g}+A_{2 g}+B_{1 g}+B_{2 g}+A_{1 u}+A_{2 u}+B_{2 u} \text {. }
$$

On a cherché quelles raies vibroniques pouvaient être associées aux raies à zéro phonon déjà sûrement identifiées. Les résultats sont consignés sur le tableau III. On voit qu'il y en a peu.

La démarche inverse qui consiste à rechercher les raies à zéro phonon $\Gamma_{1}$ et $\Gamma_{5}$ en examinant systématiquement si, aux différentes raies des spectres $\sigma$ et $\pi$, on peut associer tout ou partie des structures vibrationnelles $\sigma$ ou $\pi$ attendues, conduit à des résultats qu'il convient d'exploiter avec prudence. Ainsi la raie à $4578 \AA(\sigma)\left(21837 \mathrm{~cm}^{-1}\right)$ semble liée aux raies $4568 \AA(\sigma)\left(21884 \mathrm{~cm}^{-1}\right), 4554 \AA(\sigma)$ $\left(21951 \mathrm{~cm}^{-1}\right)$ et $4543 \AA(\sigma)\left(22006 \mathrm{~cm}^{-1}\right)$ qui sont respectivement à 47,114 et $169 \mathrm{~cm}^{-1}$ de celle-ci. Ce pourrait être une raie à zéro phonon $\Gamma_{1}$.

5. Conclusion. - L'interprétation rigoureuse $\mathrm{du}$ spectre d'absorption de $\mathrm{ThBr}_{4}: \mathrm{U}^{4+}$ de nature vibronique passe par l'identification d'un nombre maxi- mum de raies à zéro phonon. Cela nécessite l'accumulation de nombreux résultats complémentaires à ceux obtenus en analysant simplement la lumière absorbée par ces monocristaux. Nous avons déjà mis en œuvre des techniques qui en fournissent. D'autres sont en cours d'exploitation.

Compte tenu de la complexité extrême des niveaux associés à la configuration $5 \mathrm{f}^{2}$, due essentiellement à l'enchevêtrement des états $\mathrm{J}$, il est probable que toutes les indexations des spectres de $\mathrm{U}^{4+}$, notamment en basse symétrie, qui ont été proposées jusqu'à aujourd'hui soient à réviser. Les conclusions qui en découlent aussi.

Ce travail s'inscrit dans la perspective de l'étude de la nature des interactions des ions $\mathrm{M}^{4+} 5 \mathrm{f}^{q}(q=1$, $2,3,4)$ dans les halogénures de thorium ou d'autres composés par spectroscopie optique à haute résolution.

Remerciements. - Nous tenons à remercier les Professeurs Chapelle, Mattler, Mathieu et Pick, responsables des différents laboratoires où nous avons pu effectuer nos mesures.

\section{Bibliographie}

[1] Genet, M., Delamoye, P., Edelstein, N. and Conway, J., J. Chem. Phys. 67 (1977) 1620.

[2] Hecht, H. G. and Gruber, J. B., J. Chem. Phys. 60 (1974) 4872.

[3] Mackey, D. J., Runciman, W. A. and Vance, E. R., Phys. Rev. B 11 (1975) 211.
[4] Sofen, S. R., Abu-Dari, K., Freyberg, D. P. and Raymond, K. N. (soumis au J. Am. Chem. Soc.)

[5] Publication en rapport interne IPNO (Orsay) à paraître.

[6] Delwaulle, M. L. et Françors, M. F., J. Physique Radium 7 (1946) 15. 